\title{
Alteraciones del sueño más frecuentes en las personas en diálisis
}

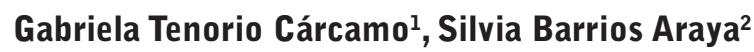

${ }^{1}$ Enfermera. Postítulo de Enfermería del Adulto con problemas renales. Escuela de Enfermería. Pontificia Universidad Católica de Chile. Santiago-Chile

${ }^{2}$ Enfermera. Profesor Asociado. Escuela de Enfermería Pontificia Universidad Católica de Chile. Presidenta de la Sociedad Latinoamericana de Enfermería Nefrológica (SLAEN). Santiago-Chile

\section{Resumen}

Introducción: La persona con enfermedad renal crónica en terapia dialítica presenta alteraciones en diferentes dimensiones de su vida, entre ellos el sueño, considerado una de las necesidades biológicas más importantes del organismo.

Objetivo: Conocer las principales alteraciones del sueño en pacientes con enfermedad renal crónica en tratamiento dialítico, los factores que influyen en su aparición y cuidados de enfermería.

Método: Revisión narrativa de la literatura. Los criterios de inclusión fueron estudios primarios y revisiones sistemáticas, tanto cualitativos como cuantitativos, sobre alteraciones del sueño en pacientes adultos con enfermedad renal crónica en terapia dialítica. Se consultaron las bases de datos Medline, Scielo y Epistemonikos.

Resultados: La prevalencia de trastornos del sueño es alta en personas con enfermedad renal crónica que se someten a diálisis, las principales son síndrome de piernas inquietas y síndrome de apnea obstructiva del sueño. En el primero, el principal factor causal es el déficit de hierro y disfunción de dopamina, mientras que en el segundo son la sobrecarga de volumen y estrechez de vías respiratorias. El manejo responde a medidas farmacológicas como administración de hierro, agentes

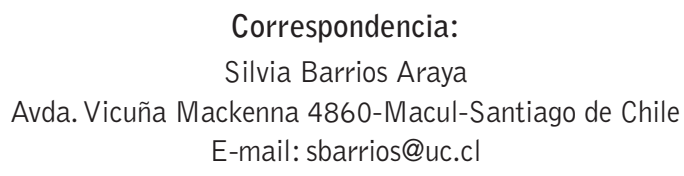

dopaminérgicos y uso de CPAP, además de medidas no farmacológicas que son transversales a ambos, como higiene del sueño.

Conclusión: Las alteraciones del sueño en los pacientes en diálisis claramente repercuten en su calidad de vida, por lo que es un aspecto en que los profesionales de salud debiesen adquirir conocimientos y otorgar cuidados de enfermería específicos para prevenir, atenuar síntomas y evitar complicaciones.

PALABRAS CLAVE: disomnias; trastornos del sueño; diálisis renal; atención de enfermería

\section{Most frequent sleep disorders in dialysis patients}

\section{Abstract}

Introduction: The person with chronic kidney disease in dialysis therapy has alterations in different dimensions of life, including sleep, considered one of the most important biological needs of the organism.

Objective: To know the main sleep alterations in patients with chronic kidney disease in dialysis treatment, predisposing factors and nursing care.

Method: Narrative review of the literature. The inclusion criteria were primary studies and systematic reviews, both qualitative and quantitative, on sleep alterations in adult patients with chronic kindey disease in dialysis therapy. The Medline, Scielo and Epistemonikos databases were consulted. 
Results: The prevalence of sleep disorders is high in people with chronic kidney disease who undergo dialysis, the main ones being restless legs syndrome and obstructive sleep apnea syndrome. In the first, the main causative factor was iron deficiency and dysfunction of dopamine, while the second was the volume overload and narrow airways. The therapeutic approach responds to pharmacological measures such as iron administration, dopaminergic agents and the use of CPAP, as well as non-pharmacological measures, transversal to both such as sleep hygiene.

Conclusion: Sleep disturbances in dialysis patients clearly affect their quality of life, being an aspect in which health professionals should acquire knowledge and provide specific nursing care to prevent, attenuate symptoms and avoid complications.

KEYWORDS: disomnias; sleep disorders; renal dialysis; nursing care.

\section{Introducción}

La hemodiálisis (HD) es la terapia de reemplazo renal mayormente utilizada en los pacientes con enfermedad renal crónica (ERC) a nivel mundial y en Chile ${ }^{1}$. A pesar de que permite prolongar la vida de la persona, impacta en diferentes ámbitos de la vida del paciente: cambios de alimentación, dinámica familiar, económicas, trastornos del sueño, presencia de síntomas físicos, entre otros 2,3 .

El sueño es una necesidad biológica prioritaria para todo ser, en los pacientes con ERC los problemas relacionados con el sueño se consideran de importancia clínica, dado que generan estrés y afectan negativamente la calidad de vida ${ }^{3,4}$. De los múltiples problemas a los que se ven enfrentados los pacientes en diálisis, los más reportados son los trastornos del sueño, que alcanzan una prevalencia entre el $60 \%$ y $80 \%^{3}$. Una revisión sistemática con 17 estudios, reveló que los problemas del sueño eran uno de los problemas más comunes en esta población ${ }^{5}$. Frente a este escenario, surge el interés de conocer cuáles son los problemas de sueño más prevalentes y qué estrategias de abordaje son efectivas para enfermería.

El objetivo de esta revisión es conocer las principales alteraciones del sueño en pacientes con ERC en tratamiento dialítico, los factores que influyen en su aparición y los cuidados de enfermería.

\section{Material y Método}

Se realizó una revisión narrativa de la literatura. Los criterios de inclusión fueron estudios primarios y revisiones sistemáticas, sobre alteraciones del sueño en pacientes adultos con ERC en terapia dialítica.

Para la revisión, se consultaron las bases de datos Medline, Scielo, Cochrane y Epistemonikos. La estrategia de búsqueda fue ["sleep disturbances" OR" $d$ yssomnias"] AND "renal dialysis", acotada a los últimos 10 años (del 01/01/2006 al 31/12/2016), sin restricción de idioma. Además, se agregó una búsqueda dirigida a terapias cognitiva-conductual de las alteraciones del sueño, cuya estrategia de búsqueda fue ["non-pharmacological interventions"] AND ["insomnia" OR "dyssomnias"].

Se realizó un primer cribado de los registros de los artículos devueltos por las bases de datos, con una lectura de título y abstract, para detectar duplicidades y realizar una primera selección. Tras la misma, se accedió al texto completo de los artículos seleccionados y se realizó su lectura para determinar su idoneidad, descartándose 2 por no tener acceso real y 2 por presentar resultados no concluyentes.

\section{Resultados}

Finalmente se seleccionaron 43 artículos para realizar la síntesis narrativa (la Figura 1 muestra el diagrama de flujo de la selección de artículos y en las tablas 1 a 5 los documentos revisados clasificados por tema). De cada artículo se realizó una ficha resumen, donde se organizó la información de acuerdo a las alteraciones del sueño, considerando su descripción, manifestaciones, prevalencia, factores de riesgo, diagnóstico y complicaciones.

A partir de la información compilada se realizó una síntesis narrativa, agrupada por los siguientes aspectos.

- Síndrome de piernas inquietas y movimiento periódico de las extremidades.

- Apnea obstructiva del sueño.

- Somnolencia diurna excesiva.

- Insomnio. 


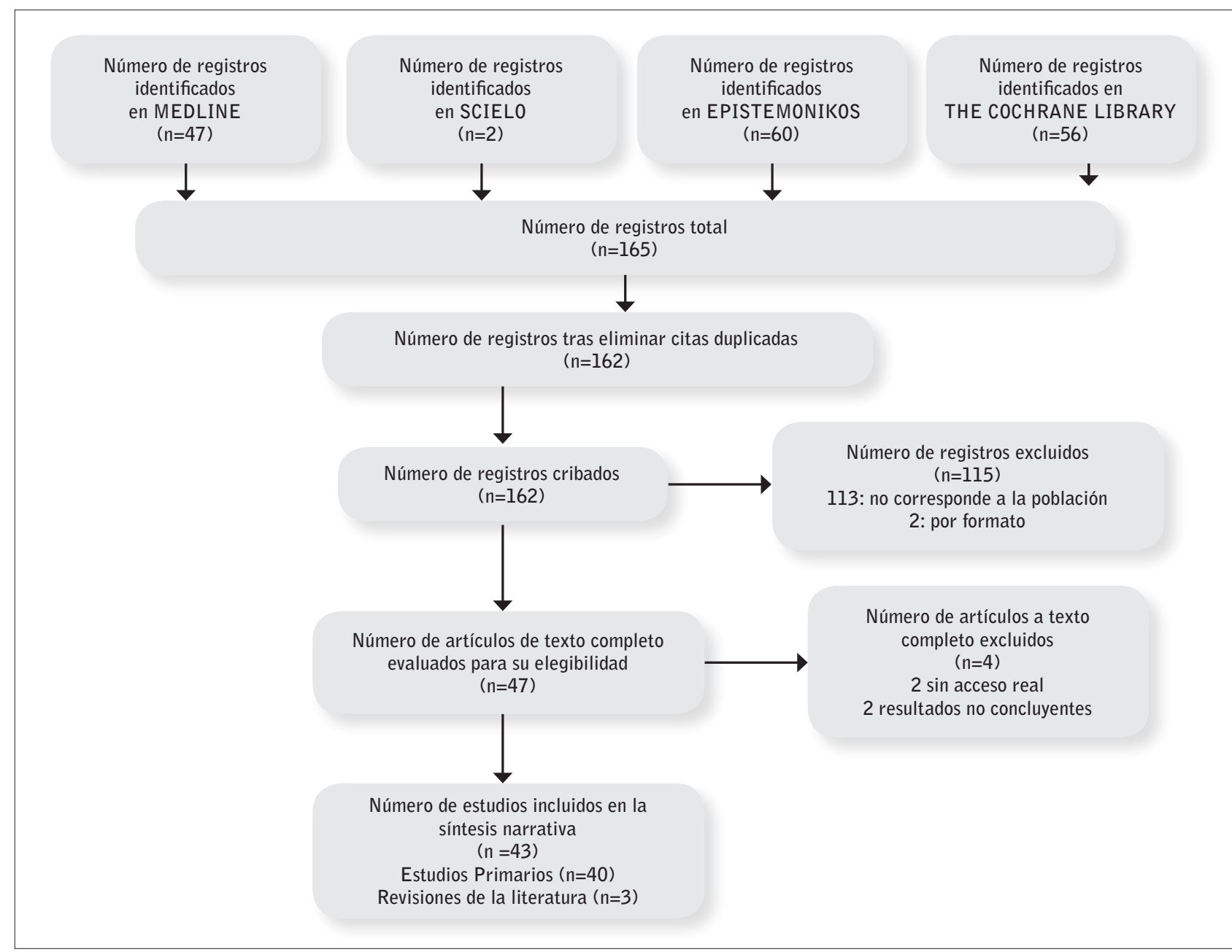

Figura 1. Diagrama de flujo de la selección de artículos.

Tabla 1. Artículos revisados sobre síndrome de piernas inquietas.

\begin{tabular}{|c|c|c|c|c|}
\hline AUTOR, AÑo & $\begin{array}{l}\text { POBLACIÓN Y } \\
\text { MUESTRA }\end{array}$ & OBJETIVOS & CONCLUSIONES & TIPO \\
\hline $\begin{array}{l}\text { Demircioğlu T, } \\
\text { Kavadar G, Esen Öre } \\
\text { Ö et al; } 2015^{9} \text {. }\end{array}$ & $\begin{array}{l}118 \text { pacientes en } \\
\text { HD. }\end{array}$ & $\begin{array}{l}\text { Determinar la prevalencia de SPI } \\
\text { en pacientes en hemodiálisis re- } \\
\text { gular, su relación con la calidad de } \\
\text { vida de los pacientes, datos sociode- } \\
\text { mográficos y de laboratorio. }\end{array}$ & $\begin{array}{l}\text { Pacientes en HD con SPI presen- } \\
\text { tan menor calidad de vida física. } \\
\text { No hay asociación significativa en- } \\
\text { tre SPI y ninguno de los siguientes } \\
\text { factores: edad, sexo, duración de la } \\
\text { diálisis y exámenes de laboratorio } \\
\text { (ferritina y PTH). }\end{array}$ & EP \\
\hline $\begin{array}{l}\text { Wali S, Alkhouli A; } \\
2015^{10} \text {. }\end{array}$ & $\begin{array}{l}355 \text { pacientes en } \\
\text { HD. }\end{array}$ & $\begin{array}{l}\text { Determinar la prevalencia del SPI } \\
\text { en pacientes sauditas con IRC en } \\
\text { hemodiálisis. }\end{array}$ & $\begin{array}{l}\text { La prevalencia fue cerca del } 20 \% \text {. } \\
\text { SPI se asoció significativamente } \\
\text { con SAOS y SDE. No mostró corre- } \\
\text { lación con la adecuación de HD, la } \\
\text { cronicidad, frecuencia y la duración } \\
\text { de la hemodiálisis. Un IMC mayor, } \\
\text { uso de aspirina y anticoagulantes } \\
\text { aparecen como factor de riesgo } \\
\text { para desarrollar SPI. }\end{array}$ & EP \\
\hline
\end{tabular}




\begin{tabular}{|c|c|c|c|c|}
\hline AUTOR, AÑo & $\begin{array}{l}\text { POBLACIÓN Y } \\
\text { MUESTRA }\end{array}$ & OBJETIVOS & CONCLUSIONES & TIPO \\
\hline Salman SM; $2011^{11}$. & $\begin{array}{l}123 \text { pacientes en } \\
\text { HD. }\end{array}$ & $\begin{array}{l}\text { Determinar la prevalencia de SPI } \\
\text { en pacientes HD y para encontrar la } \\
\text { posible correlación entre la presen- } \\
\text { cia de SPI y demográfica, clínica, y } \\
\text { factores bioquímicos. }\end{array}$ & $\begin{array}{l}\text { No encontraron factores estadísti- } \\
\text { camente significativos que favorez- } \\
\text { can el desarrollo de SPI. La alta } \\
\text { prevalencia de SPI entre pacientes } \\
\text { en HD requiere una atención } \\
\text { cuidadosa y un diagnóstico correcto } \\
\text { puede conducir a una mejor terapia } \\
\text { y una mejor calidad de vida. }\end{array}$ & EP \\
\hline $\begin{array}{l}\text { Rafie S, Jafari M, } \\
\text { Azizi M, Bahadoram } \\
\text { M; } 2016^{15} \text {. }\end{array}$ & $\begin{array}{l}137 \text { pacientes en } \\
\text { HD. }\end{array}$ & $\begin{array}{l}\text { Determinar la prevalencia, los } \\
\text { factores de riesgo y la gravedad } \\
\text { de SPI en nuestra población de } \\
\text { hemodiálisis. }\end{array}$ & $\begin{array}{l}\text { Las alteraciones neurodege- } \\
\text { nerativas, menores niveles de } \\
\text { dopamina, mayor estado oxidativo } \\
\text { y neuropatía diabética avanzada } \\
\text { podrían exacerbar los síntomas } \\
\text { de SPI. Considera a la diabetes y } \\
\text { neuropatía como factor de riesgo } \\
\text { de presentar SPI. }\end{array}$ & EP \\
\hline $\begin{array}{l}\text { Lindner A, Fornadi K, } \\
\text { Lazar AS, Czira ME; } \\
2012^{17} \text {. }\end{array}$ & $\begin{array}{l}150 \text { pacientes } \\
\text { (trasplantados-HD). }\end{array}$ & $\begin{array}{l}\text { Determinar la prevalencia de esta } \\
\text { enfermedad en una muestra de } \\
\text { pacientes trasplantados y en lista de } \\
\text { espera hemodializados. }\end{array}$ & $\begin{array}{l}\text { La prevalencia de MPE es mayor } \\
\text { en pacientes en HD (esperando } \\
\text { trasplante) que en los trasplanta- } \\
\text { dos. EI MPE se relaciona directa- } \\
\text { mente con mayor riesgo cardiovas- } \\
\text { cular y cerebrovascular. }\end{array}$ & EP \\
\hline $\begin{array}{l}\text { Soumeila I, Keita S, } \\
\text { Elhassani A, Sidibe } \\
\text { M, et al; } 2015^{20} \text {. }\end{array}$ & 84 pacientes en HD. & $\begin{array}{l}\text { Determinar la frecuencia del SPI } \\
\text { en población de hemodiálisis e } \\
\text { identificar los factores asociados } \\
\text { con el SPI. }\end{array}$ & $\begin{array}{l}\text { Prevalencia de } 41 \% \text {. HTA y déficit } \\
\text { de hierro se asocia significativa- } \\
\text { mente con SPI. Niveles de Ca o } \\
\text { de P no se asociaron con SPI de } \\
\text { manera significativa. }\end{array}$ & EP \\
\hline $\begin{array}{l}\text { Mortazavi M, } \\
\text { Vahdatpour B, } \\
\text { Ghasenpour A, Taheri } \\
\text { D, et al; } 2013^{21} \text {. }\end{array}$ & 26 pacientes en HD. & $\begin{array}{l}\text { Evaluar el efecto del ejercicio } \\
\text { aeróbico sobre la severidad del SPI } \\
\text { y la calidad de vida de los pacientes } \\
\text { durante la hemodiálisis crónica. }\end{array}$ & $\begin{array}{l}\text { Sugiere el ejercicio físico aeróbico } \\
\text { como tratamiento para mejorar } \\
\text { los síntomas de SPI, sin embargo, } \\
\text { no mejora la calidad de vida del } \\
\text { paciente. }\end{array}$ & EP \\
\hline $\begin{array}{l}\text { Gade K, Blaschke S, } \\
\text { Rodenbeck A, Becker, } \\
\text { A, et al; } 2013^{22} \text {. }\end{array}$ & 52 pacientes en HD. & $\begin{array}{l}\text { Investigar si la elevación de la } \\
\text { concentración plasmática de } \\
\text { homocisteína podría asociarse con } \\
\text { la aparición de SPI en pacientes } \\
\text { en HD. }\end{array}$ & $\begin{array}{l}\text { Existe una relación significativa } \\
\text { entre niveles de homocisteína plas- } \\
\text { mática y SPI. La calidad de vida se } \\
\text { ve alterada en pacientes con SPI. } \\
\text { La intensidad de los síntomas de } \\
\text { SPI y altos niveles de PTH se aso- } \\
\text { cian con mala calidad del sueño. }\end{array}$ & EP \\
\hline $\begin{array}{l}\text { Al-Jahdali HH, Al- } \\
\text { Qadhy WA, Khogeer } \\
\text { HA, Al-Hejaili FF; } \\
2009^{24} \text {. }\end{array}$ & $\begin{array}{l}227 \text { pacientes } \\
\text { (HD-población } \\
\text { general). }\end{array}$ & $\begin{array}{l}\text { Averiguar la prevalencia de SPI en } \\
\text { pacientes sauditas con ERC en HD. }\end{array}$ & $\begin{array}{l}\text { Prevalencia del } 50 \% \text {, más frecuen- } \\
\text { te que la población general. Los } \\
\text { predictores significativos de SPI } \\
\text { fueron diabetes mellitus, consumo } \\
\text { de café, diálisis por la tarde, sexo } \\
\text { masculino y hemodiálisis. Pacien- } \\
\text { tes con SPI tenían un mayor riesgo } \\
\text { de tener insomnio y SDE. }\end{array}$ & EP \\
\hline $\begin{array}{l}\text { Giannaki CD, Sakkas } \\
\text { GK, Karatzaferi C, } \\
\text { Hadjigeorgiou GM, et } \\
\text { al; } 2013^{28} \text {. }\end{array}$ & 32 pacientes en HD. & $\begin{array}{l}\text { Comparar los cambios entre los } \\
\text { grupos en la mejora de los síntomas } \\
\text { SPI en pacientes con HD con SPI. }\end{array}$ & $\begin{array}{l}\text { La realización de ejercicio aeróbi- } \\
\text { co y el uso de agonistas de dopami- } \\
\text { na fueron eficaces para disminuir } \\
\text { los síntomas de SPI. Ambos me- } \\
\text { joraron la calidad de vida, aunque } \\
\text { solo los agonistas dopaminérgicos } \\
\text { mejoraron la calidad de sueño. }\end{array}$ & EP \\
\hline
\end{tabular}




\begin{tabular}{|c|c|c|c|c|}
\hline AUTOR, AÑO & $\begin{array}{l}\text { POBLACIÓN Y } \\
\text { MUESTRA }\end{array}$ & OBJETIVOS & CONCLUSIONES & TIPO \\
\hline $\begin{array}{l}\text { Razazian N, Azimi } \\
\text { H, Heidarnejadian } \\
\text { J, Afshari D, et al; } \\
2015^{29} \text {. }\end{array}$ & 87 pacientes en HD. & $\begin{array}{l}\text { Comparar la eficacia de la ga- } \\
\text { bapentina versus levodopa en el } \\
\text { tratamiento de SPI. }\end{array}$ & $\begin{array}{l}\text { Ambos fueron eficaces, aunque la } \\
\text { gabapentina es más significativa. } \\
\text { Ambos mejoraron los parámetros } \\
\text { del sueño: calidad, latencia y } \\
\text { duración. }\end{array}$ & EP \\
\hline $\begin{array}{l}\text { Giannaki CD, Sakkas } \\
\text { GK, Karatzaferi C, } \\
\text { Hadgigeorgiou GM, et } \\
\text { al; } 2011^{30} \text {. }\end{array}$ & 70 pacientes en HD. & $\begin{array}{l}\text { Investigar si los pacientes de HD } \\
\text { con SPI, en comparación con } \\
\text { aquellos sin el síndrome, muestran } \\
\text { evidencia de reducción de la calidad } \\
\text { de vida, rendimiento físico global y } \\
\text { capacidad funcional, así como nue- } \\
\text { vas alteraciones en los índices de } \\
\text { composición corporal y característi- } \\
\text { cas musculares. }\end{array}$ & $\begin{array}{l}\text { Se evidencia atrofia muscular } \\
\text { del muslo en pacientes con SPI. } \\
\text { La calidad del sueño y depresión } \\
\text { aparecen significativamente en el } \\
\text { grupo SPI. El componente mental } \\
\text { del cuestionario de calidad de vida } \\
\text { está estadísticamente alterado en } \\
\text { pacientes con SPI. }\end{array}$ & EP \\
\hline $\begin{array}{l}\text { Stolic RV, Trajkovic } \\
\text { GZ, Jekic D, Sovtic } \\
\text { SR, et al; } 2014^{32} \text {. }\end{array}$ & $\begin{array}{l}204 \text { pacientes en } \\
\text { HD. }\end{array}$ & $\begin{array}{l}\text { Determinar las características y } \\
\text { los parámetros de supervivencia en } \\
\text { pacientes en HD con SPI. }\end{array}$ & $\begin{array}{l}\text { Sugiere que un mayor IMC es } \\
\text { factor de riesgo de desarrollar } \\
\text { SPI. Sugiere que la diabetes y un } \\
\text { bajo Kt/V en pacientes con SPI son } \\
\text { factores de riesgo de mortalidad. }\end{array}$ & EP \\
\hline $\begin{array}{l}\text { Giannaki CD, Zigoulis } \\
\text { P, Karatzaferi C, } \\
\text { Hadjigeorgiou GM, et } \\
\text { al; } 2013^{33} \text {. }\end{array}$ & 19 pacientes en HD. & $\begin{array}{l}\text { Determinar la prevalencia del MPE } \\
\text { en pacientes trasplantados y en lista } \\
\text { de espera hemodializados. }\end{array}$ & $\begin{array}{l}\text { Pacientes con MPE presentan un } \\
\text { aumento significativo de la masa } \\
\text { del ventrículo izquierdo y por ende, } \\
\text { mayor riesgo cardiovascular. }\end{array}$ & EP \\
\hline $\begin{array}{l}\text { Loewen A, Siemens A, } \\
\text { Hanly P; } 2009^{42} \text {. }\end{array}$ & $\begin{array}{l}30 \text { pacientes } \\
\text { (HD-población } \\
\text { general). }\end{array}$ & $\begin{array}{l}\text { Evaluar la calidad del sueño en los } \\
\text { pacientes con ERC que son recién } \\
\text { diagnosticados con SAOS y determi- } \\
\text { nar la contribución adicional de SPI } \\
\text { a la interrupción del sueño. }\end{array}$ & $\begin{array}{l}\text { Pacientes en HD presentan mayor } \\
\text { prevalencia de SPI, además de } \\
\text { un sueño total más corto, menor } \\
\text { eficiencia del sueño, mayor movi- } \\
\text { miento de extremidades. }\end{array}$ & EP \\
\hline $\begin{array}{l}\text { Jaber BL, Schiller B, } \\
\text { Burkart JM, Daoui R; } \\
2011^{52} \text {. }\end{array}$ & $\begin{array}{l}235 \text { pacientes en } \\
\text { HD. }\end{array}$ & $\begin{array}{l}\text { Evaluar el efecto a largo plazo de } \\
\text { la HD sobre la prevalencia y grave- } \\
\text { dad de SPI, también las alteracio- } \\
\text { nes del sueño. }\end{array}$ & $\begin{array}{l}\text { La HD domiciliaria se asocia con } \\
\text { una mejoría a largo plazo en la } \\
\text { prevalencia y la gravedad del SPI y } \\
\text { diferentes alteraciones del sueño. }\end{array}$ & EP \\
\hline $\begin{array}{l}\text { Unruh ML, Buysse } \\
\text { DJ , Dew MA, Evans } \\
\text { IV, et al; } 2006^{54} \text {. }\end{array}$ & $\begin{array}{l}1041 \text { pacientes en } \\
\text { HD. }\end{array}$ & $\begin{array}{l}\text { Examinar los factores predictivos } \\
\text { de la calidad del sueño entre los } \\
\text { participantes y determinar los } \\
\text { factores relacionados en la calidad } \\
\text { del sueño. Además, examinar la } \\
\text { relación entre la calidad del sueño y } \\
\text { la supervivencia. }\end{array}$ & $\begin{array}{l}\text { Una mala calidad de sueño se rela- } \\
\text { ciona significativamente con raza } \\
\text { negra, mayor fosfemia, tabaquismo } \\
\text { actual, uso de benzodiazepinas y } \\
\text { SPI. Como consecuencia se obtuvo } \\
\text { disminución en la calidad de vida } \\
\text { física y mental, así como también, } \\
\text { en vitalidad y dolor corporal. Una } \\
\text { disminución en la calidad del sueño } \\
\text { durante el primer año en diálisis se } \\
\text { asoció con una supervivencia más } \\
\text { corta. }\end{array}$ & EP \\
\hline $\begin{array}{l}\text { Seerapani Gopaluni, } \\
\text { Mohamed Sherif, } \\
\text { Naim A Ahmadouk; } \\
2016^{18} \text {. }\end{array}$ & $\begin{array}{l}9 \text { estudios; } 200 \\
\text { pacientes en HD. }\end{array}$ & $\begin{array}{l}\text { Analizar críticamente los beneficios } \\
\text { y seguridad de las diversas opciones } \\
\text { de tratamiento utilizadas en SPI } \\
\text { en personas con ERC y aquellas en } \\
\text { diálisis. }\end{array}$ & $\begin{array}{l}\text { Las intervenciones farmacológicas } \\
\text { y los programas de ejercicio intra- } \\
\text { dialítico tienen efectos inciertos en } \\
\text { el SPI en pacientes en hemodiá- } \\
\text { lisis. El ejercicio de resistencia } \\
\text { aeróbica y el ropinirol pueden ser } \\
\text { intervenciones adecuadas. }\end{array}$ & $\mathrm{RL}$ \\
\hline
\end{tabular}

SPI: síndrome de piernas inquietas, EP: estudio primario, ERC: enfermedad renal crónica, HD: hemodiálisis; IMC: índice de masa corporal, IRC: insuficiencia renal crónica, MPE: movimiento periódico de extremidades, RL: revisión de la literatura, SAOS: síndrome de apnea obstructiva del sueño, SDE: somnolencia diurna excesiva. 
Tabla 2. Artículos revisados sobre síndrome de apnea obstructiva del sueño.

\begin{tabular}{|c|c|c|c|c|}
\hline AUTOR, AÑo & $\begin{array}{l}\text { POBLACIÓN Y } \\
\text { MUESTRA }\end{array}$ & OBJETIVOS & CONCLUSIONES & TIPO \\
\hline $\begin{array}{l}\text { Forni Ogna V, Ogna A, } \\
\text { Pruijm M, Bassi I, et } \\
\text { al; } 2015^{34} \text {. }\end{array}$ & $\begin{array}{l}125 \text { pacientes en } \\
\text { HD. }\end{array}$ & $\begin{array}{l}\text { Evaluar la prevalencia de SAOS y } \\
\text { el rendimiento de las herramientas } \\
\text { de detección disponibles. }\end{array}$ & $\begin{array}{l}\text { La prevalencia de SAOS modera- } \\
\text { da-severa en esta población fue } \\
56 \%, \text { y } 31 \% \text { con SAOS severa, } \\
\text { pero sólo } 19 \% \text { de todos los } \\
\text { pacientes con SAOS habían sido } \\
\text { previamente diagnosticados y } 10 \% \\
\text { fue tratado. Se utiliza la polisom- } \\
\text { nografía como método diagnóstico, } \\
\text { siendo éste más preciso que los } \\
\text { cuestionarios. }\end{array}$ & EP \\
\hline $\begin{array}{l}\text { Ogna A, Forni-Ogna V, } \\
\text { Mihalache A, Prujim } \\
\text { M, et al; } 2015^{35} \text {. }\end{array}$ & 17 pacientes en HD. & $\begin{array}{l}\text { Comprobar que una menor sobre- } \\
\text { carga de líquidos después de HD } \\
\text { resultaría en menor volumen de } \\
\text { desplazamiento nocturno y, por tan- } \\
\text { to, una menor gravedad de SAOS. }\end{array}$ & $\begin{array}{l}\text { Una menor sobrecarga de volumen } \\
\text { se relaciona significativamente con } \\
\text { un menor AHÍ. El AHÍ es significa- } \\
\text { tivamente menor post-hemodiálisis. }\end{array}$ & EP \\
\hline $\begin{array}{l}\text { Roumelioti ME, } \\
\text { Buyysse DJ, Sanders } \\
\text { MH, Strollo P; } \\
2011^{36} \text {. }\end{array}$ & 388 personas. & $\begin{array}{l}\text { Describir y comparar prevalencia, } \\
\text { gravedad y patrones de SAOS y } \\
\text { SDE entre pacientes con HD e } \\
\text { individuos de la comunidad sin } \\
\text { enfermedad renal conocida. }\end{array}$ & $\begin{array}{l}\text { La probabilidad de desarrollar } \\
\text { SAOS fue más alta para el grupo } \\
\text { de HD que el control (sin HD). }\end{array}$ & EP \\
\hline $\begin{array}{l}\text { Beecroft JM, } \\
\text { Hoffstein V, Pierratos } \\
\text { A, Chan CT; } 2007^{38} \text {. }\end{array}$ & 85 pacientes en HD. & $\begin{array}{l}\text { Comparar las dimensiones de la } \\
\text { faringe en pacientes con y sin ERC, } \\
\text { subdividida personas con y sin } \\
\text { SAOS, para comprobar si ERC se } \\
\text { asocia con una vía aérea superior } \\
\text { estrechada. }\end{array}$ & $\begin{array}{l}\text { El área faríngea es menor en pa- } \\
\text { cientes en HD que en la población } \\
\text { general, lo que repercute en la } \\
\text { calidad del sueño por SAOS. }\end{array}$ & EP \\
\hline $\begin{array}{l}\text { Beecroft JM, } \\
\text { Pierratos A, Hanly } \\
\text { PJ; } 2009^{39} \text {. }\end{array}$ & $\begin{array}{l}456 \text { pacientes } \\
\text { (HD-Población } \\
\text { gral). }\end{array}$ & $\begin{array}{l}\text { Determinar si la presentación de } \\
\text { SAOS en pacientes con ERC difiere } \\
\text { de la presentación estereotipada en } \\
\text { la población general. }\end{array}$ & $\begin{array}{l}\text { Los síntomas del SAOS difieren } \\
\text { entre los pacientes con HD y sin } \\
\text { ERC: menos ronquidos fuertes y } \\
\text { más trastornos del sueño. Debe } \\
\text { ser considerado para evitar el in- } \\
\text { fradiagnóstico de esta importante } \\
\text { comorbilidad. }\end{array}$ & EP \\
\hline $\begin{array}{l}\text { Tuohy C, Montez-Rath } \\
\text { M, Turakhia M, Chang } \\
\text { TI et al; } 2016^{41} \text {. }\end{array}$ & $\begin{array}{l}184.217 \text { pacientes } \\
\text { en HD. }\end{array}$ & $\begin{array}{l}\text { Comprobar que SAOS está asociado } \\
\text { a mayor mortalidad por todas las } \\
\text { causas y eventos cardiovasculares } \\
\text { en pacientes añosos con ERC. }\end{array}$ & $\begin{array}{l}\text { Sugiere que SAOS puede no ser } \\
\text { un factor de riesgo tan importante } \\
\text { para eventos cardiovasculares en } \\
\text { pacientes mayores con ERC. }\end{array}$ & EP \\
\hline $\begin{array}{l}\text { Tang SA, Lam B, } \\
\text { Yao TJ, Leung WS; } \\
2010^{44} \text {. }\end{array}$ & 93 pacientes en PD. & $\begin{array}{l}\text { Estudiar el SAOS en pacientes en } \\
\text { DP. }\end{array}$ & $\begin{array}{l}\text { Los bajos niveles de saturación de } \\
\text { oxígeno en pacientes con DP es un } \\
\text { predictor de mortalidad y eventos } \\
\text { cardiovasculares, así como también } \\
\text { el aumento de AHÍ. Se establece } \\
\text { que SAOS es un factor indepen- } \\
\text { diente de mortalidad por todas las } \\
\text { causas. }\end{array}$ & EP \\
\hline $\begin{array}{l}\text { Tang SA, Lam B, Lai } \\
\text { AS, Pang CB; } 2009^{45} \text {. }\end{array}$ & 38 pacientes en PD. & $\begin{array}{l}\text { Comprobar que la DPN versus la } \\
\text { DPCA promueve una mejor depura- } \\
\text { ción y limpieza de líquidos el sueño, } \\
\text { lo que puede estar asociado con } \\
\text { mejora de SAOS. }\end{array}$ & $\begin{array}{l}\text { La mejoría en los síntomas del } \\
\text { SAOS en DP se asocia con un me- } \\
\text { jor aclaramiento urémico y de flui- } \\
\text { dos, además de una reducción de la } \\
\text { congestión de las vías respiratorias } \\
\text { superiores durante el sueño. }\end{array}$ & EP \\
\hline
\end{tabular}




\begin{tabular}{|c|c|c|c|c|}
\hline AUTOR, AÑo & $\begin{array}{l}\text { POBLACIÓN Y } \\
\text { MUESTRA }\end{array}$ & OBJETIVOS & CONCLUSIONES & TIPO \\
\hline $\begin{array}{l}\text { Dos Reis Santos } \\
\text { I, Damaga AR, de } \\
\text { Carvalho Aguiar I, } \\
\text { Oliveira EF, et al; } \\
2013^{46} \text {. }\end{array}$ & $\begin{array}{l}214 \text { pacientes en } \\
\text { HD. }\end{array}$ & $\begin{array}{l}\text { Comprobar que el aumento de peso } \\
\text { debido a la sobrecarga de volumen } \\
\text { observada durante el período } \\
\text { interdiálisis influirá en el grado de } \\
\text { colapso de la vía aérea superior. }\end{array}$ & $\begin{array}{l}\text { Se describe el material y métodos } \\
\text { de un estudio con el fin de corrobo- } \\
\text { rar los factores de riesgo asociados } \\
\text { a los trastornos del sueño. En } \\
\text { esta publicación, no se muestran } \\
\text { resultados dado que es la primera } \\
\text { parte del estudio. }\end{array}$ & EP \\
\hline $\begin{array}{l}\text { Masuda T, Murata M, } \\
\text { Honma S, Iwazu Y et } \\
\text { al; } 2011^{49} \text {. }\end{array}$ & 94 pacientes en HD. & $\begin{array}{l}\text { Realizar un seguimiento a los } \\
\text { pacientes en HD con SAOS en } \\
\text { relación con la mortalidad. }\end{array}$ & $\begin{array}{l}\text { EI SAOS es un factor de ries- } \\
\text { go independiente para eventos } \\
\text { cardiovasculares y mortalidad en } \\
\text { pacientes con HD. }\end{array}$ & EP \\
\hline $\begin{array}{l}\text { Mavanur M, Sanders } \\
\text { M, Rnruh M; } 2010^{31} \text {. }\end{array}$ & $\begin{array}{l}\text { Pacientes en HD, } \\
\text { DP y trasplantados. }\end{array}$ & $\begin{array}{l}\text { Estimar la prevalencia y factores } \\
\text { de riesgo asociados al SAOS en } \\
\text { pacientes en diálisis. }\end{array}$ & $\begin{array}{l}\text { La sobrecarga de volumen y } \\
\text { uremia podrían contribuir a de- } \\
\text { sarrollar SAOS. Se evidencia una } \\
\text { reducción de la gravedad de SAOS } \\
\text { en pacientes trasplantados, con DP } \\
\text { nocturna y HD nocturna. }\end{array}$ & $\mathrm{RL}$ \\
\hline
\end{tabular}

SAOS. Síndrome de apnea obstructiva del sueño, AHÍ: índice de apnea-hipoapnea, DP: diálisis peritoneal, DPCA: diálisis peritoneal contínua ambulatoria, DPN: diálisis peritoneal nocturna, EP: estudio primario, ERC: enfermedad renal crónica, HD: hemodiálisis, RL: revisión de la literatura, SDE: somnolencia diurna excesiva.

Tabla 3. Artículos revisados sobre insomnio.

\begin{tabular}{|c|c|c|c|c|}
\hline AUTOR, AÑo & $\begin{array}{l}\text { POBLACIÓN Y } \\
\text { MUESTRA }\end{array}$ & OBJETIVOS & CONCLUSIONES & TIPO \\
\hline $\begin{array}{l}\text { Malaki M, Mortazavi } \\
\text { FS, Moazemi S, } \\
\text { Shoaran M, et al; } \\
2012^{55} \text {. }\end{array}$ & 26 pacientes en HD. & $\begin{array}{l}\text { Estimar la incidencia de insomnio } \\
\text { y SPI como causa de insomnio en } \\
\text { pacientes en HD. }\end{array}$ & $\begin{array}{l}\text { Existe una fuerte relación entre } \\
\text { los niveles de hemoglobina y la du- } \\
\text { ración de la terapia de reemplazo } \\
\text { renal con insomnio. }\end{array}$ & EP \\
\hline $\begin{array}{l}\text { El Harraki R, Abda N, } \\
\text { Bentata Y, Haddiya I; } \\
2014^{57} \text {. }\end{array}$ & 93 en HD. & $\begin{array}{l}\text { Determinar la prevalencia del } \\
\text { insomnio y los factores } \\
\text { asociados a ella. }\end{array}$ & $\begin{array}{l}\text { La edad avanzada, sexo femenino, } \\
\text { mayor tiempo en diálisis, BUN alto } \\
\text { prediálisis, dolor crónico, depresión } \\
\text { e hipertensión surgen como fac- } \\
\text { tores de riesgo en la aparición de } \\
\text { insomnio. }\end{array}$ & EP \\
\hline $\begin{array}{l}\text { Zou C, Yang L, Wu Y, } \\
\text { Su G, et al; } 2015^{63} \text {. }\end{array}$ & 63 pacientes en HD. & $\begin{array}{l}\text { Evaluar la factibilidad y aceptabi- } \\
\text { lidad de acupuntura auricular en } \\
\text { puntos específicos versus puntos } \\
\text { de acupuntura no específicos para } \\
\text { el tratamiento de pacientes con } \\
\text { insomnio en HD. }\end{array}$ & $\begin{array}{l}\text { Al comparar acupuntura auricular } \\
\text { en puntos específicos versus no } \\
\text { específicos como tratamiento de in- } \\
\text { somnio los resultados se muestran } \\
\text { favorables para los específicos. }\end{array}$ & EP \\
\hline
\end{tabular}

EP: estudio primario; HD: hemodiálisis. 
Tabla 4. Artículos revisados sobre somnolencia diurna excesiva.

\begin{tabular}{llll}
\hline AUTOR, AÑo & $\begin{array}{l}\text { POBLACIÓN Y } \\
\text { MUESTRA }\end{array}$ & OBJETIVOS & CoNCLUSIONES \\
$\begin{array}{l}\text { Abdel-Kader K, } \\
\text { Jhamb M, Mandich } \\
\text { LA, Yabes J, et al; }\end{array}$ & 55 pacientes en HD. & $\begin{array}{l}\text { Comprobar la fatiga, la somnolen- } \\
\text { cia y el agotamiento demostraría } \\
\text { significativa variación diurna y los } \\
\text { síntomas serían más graves en días } \\
\text { de diálisis. }\end{array}$ & $\begin{array}{l}\text { Se demuestra variabilidad diurna y } \\
\text { diaria significativa de los síntomas } \\
\text { incluyendo fatiga, somnolencia y } \\
\text { agotamiento en los pacientes en } \\
\text { diálisis. Los días de diálisis los pa- } \\
\text { cientes mostraron mayor carga de } \\
\text { síntomas (sensación de sueño, sen- } \\
\text { sación de agotamiento, dificultad } \\
\text { para concentrarse, sentirse triste, } \\
\text { no sentirse relajado, sin sentirse } \\
\text { enérgico, sintiéndose estresado y } \\
\text { sintiéndose tenso). }\end{array}$ \\
\end{tabular}

EP: estudio primario; HD: hemodiálisis.

Tabla 5.Estudios revisados sobre de mas de una alteración del sueño.

\begin{tabular}{|c|c|c|c|c|}
\hline AUTOR, AÑO & $\begin{array}{l}\text { POBLACIÓN Y } \\
\text { MUESTRA }\end{array}$ & OBJETIVOS & CONCLUSIONES & TIPO \\
\hline Al-Jahdali H; $2011^{7}$. & $\begin{array}{l}277 \text { pacientes (HD- } \\
\text { DP). }\end{array}$ & $\begin{array}{l}\text { Comparar la prevalencia de las } \\
\text { alteraciones del sueño en pacientes } \\
\text { de HD y DP. }\end{array}$ & $\begin{array}{l}\text { La prevalencia global de SAOS, } \\
\text { SPI y SDE en pacientes en DP fue } \\
\text { significativamente mayor que en } \\
\text { HD La prevalencia de insomnio fue } \\
\text { similar en ambos grupos. }\end{array}$ & EP \\
\hline $\begin{array}{l}\text { Jung HH, Lee JH, } \\
\text { Baek HJ, Kim SJ, et } \\
\text { al; } 2010^{12} \text {. }\end{array}$ & 30 pacientes en HD. & $\begin{array}{l}\text { Investigar la asociación entre los } \\
\text { trastornos del sueño y la mortalidad } \\
\text { en pacientes con ERC. }\end{array}$ & $\begin{array}{l}\text { Durante el sueño la saturación de } \\
\text { oxígeno <90\% y un mayor movi- } \\
\text { miento periódico de extremidades } \\
\text { se asoció a mayor riesgo de muer- } \\
\text { te. No hay relación significativa de } \\
\text { un bajo AHÍ con la mortalidad. }\end{array}$ & EP \\
\hline $\begin{array}{l}\text { Bastos JP, Sousa RB, } \\
\text { Nepomuceno LA, } \\
\text { Gutiérrez-Adrianzen } \\
0 A ; 2007^{23} \text {. }\end{array}$ & $\begin{array}{l}100 \text { pacientes en } \\
\text { HD. }\end{array}$ & $\begin{array}{l}\text { Investigar la influencia del cambio } \\
\text { de diálisis en la calidad del sueño } \\
\text { y las alteraciones del sueño en } \\
\text { pacientes con ERT. }\end{array}$ & $\begin{array}{l}\text { La mala calidad del sueño se } \\
\text { relaciona con el SPI y la SDE. } \\
\text { No se asocia con una diálisis más } \\
\text { eficiente. }\end{array}$ & EP \\
\hline $\begin{array}{l}\text { Elder SJ, Pisoni RL, } \\
\text { Akizawa T, Fissell R, } \\
\text { et al; } 2008^{27} \text {. }\end{array}$ & $\begin{array}{l}11.351 \text { pacientes } \\
\text { en HD. }\end{array}$ & $\begin{array}{l}\text { Investigar la prevalencia de la } \\
\text { pobre calidad del sueño y caracte- } \\
\text { rísticas del paciente que se asocian } \\
\text { a ella. }\end{array}$ & $\begin{array}{l}\text { Existe una relación entre la poli- } \\
\text { farmacia y una calidad deficiente } \\
\text { del sueño. Ejercicio físico por lo } \\
\text { menos una vez a la semana se } \\
\text { asocia con mejor calidad de sueño. } \\
\text { Una menor calidad del sueño se } \\
\text { relaciona significativamente con un } \\
\text { componente físico y mental menos } \\
\text { auspicioso. }\end{array}$ & EP \\
\hline $\begin{array}{l}\text { Roumelioti ME, } \\
\text { Argyropoulos C, } \\
\text { Buysse DJ, Nayar H, } \\
\text { et al; } 2010^{48} \text {. }\end{array}$ & 388 personas. & $\begin{array}{l}\text { Describir y comparar prevalencia, } \\
\text { gravedad y patrones de SAOS y } \\
\text { SDE entre pacientes con HD y } \\
\text { individuos de la comunidad sin } \\
\text { enfermedad renal conocida. }\end{array}$ & $\begin{array}{l}\text { La probabilidad de desarrollar } \\
\text { SAOS fue más alta para el grupo } \\
\text { de HD que el control (sin HD). La } \\
\text { SDE fue similar para los distintos } \\
\text { grupos. }\end{array}$ & EP \\
\hline
\end{tabular}




\begin{tabular}{|c|c|c|c|c|}
\hline AUTOR, AÑO & $\begin{array}{l}\text { POBLACIÓN Y } \\
\text { MUESTRA }\end{array}$ & OBJETIVOS & CONCLUSIONES & TIPO \\
\hline Al-Jahdali H; $2012^{40}$. & $\begin{array}{l}277 \text { pacientes (HD- } \\
\text { DP). }\end{array}$ & $\begin{array}{l}\text { Determinar la calidad del sueño, } \\
\text { la prevalencia de SAOS y SDE en } \\
\text { pacientes con ERC en diálisis. }\end{array}$ & $\begin{array}{l}\text { La apnea del sueño se asoció } \\
\text { significativamente con la edad, el } \\
\text { tamaño del cuello, el cambio de } \\
\text { hemodiálisis durante la tarde y la } \\
\text { noche, la obesidad, la diabetes y } \\
\text { la hipertensión. Se asoció también } \\
\text { con SPI, insomnio y SDE. }\end{array}$ & EP \\
\hline $\begin{array}{l}\text { Anand S, Johansen } \\
\text { KL, Grimes B, Kaysen } \\
\text { GA, et al; } 2013^{58} \text {. }\end{array}$ & $\begin{array}{l}1.643 \text { pacientes } \\
\text { (HD-DP). }\end{array}$ & $\begin{array}{l}\text { Comprobar que el autoreporte de } \\
\text { actividad física sería inversamente } \\
\text { correlacionada con síntomas de } \\
\text { insomnio, SPI y depresión. }\end{array}$ & $\begin{array}{l}\text { Los pacientes que informaron } \\
\text { niveles más bajos de actividad } \\
\text { física tenían más probabilidades } \\
\text { de síntomas de insomnio, SPI y } \\
\text { depresión. }\end{array}$ & EP \\
\hline $\begin{array}{l}\text { Koch BC, Nagtegaal } \\
\text { JE, Hagen EC, Van } \\
\text { der Westerlaken MM; } \\
2008^{61} \text {. }\end{array}$ & 20 pacientes en HD. & $\begin{array}{l}\text { Investigar los efectos de la } \\
\text { melatonina exógena sobre el } \\
\text { ritmo sueño-vigilia en pacientes en } \\
\text { hemodiálisis. }\end{array}$ & $\begin{array}{l}\text { Con el uso de melatonina, el } \\
\text { periodo de latencia disminuye sig- } \\
\text { nificativamente. Permite un sueño } \\
\text { más eficiente, así como también se } \\
\text { percibe una mejoría de la calidad } \\
\text { del sueño. }\end{array}$ & EP \\
\hline $\begin{array}{l}\text { Russcher M, Koch BC, } \\
\text { Nagtegaal JE, van } \\
\text { Ittersum FJ, et al; } \\
2013^{62} \text {. }\end{array}$ & 77 pacientes en HD. & $\begin{array}{l}\text { Investigar los efectos de la } \\
\text { melatonina exógena sobre el } \\
\text { ritmo sueño-vigilia en pacientes en } \\
\text { hemodiálisis. }\end{array}$ & $\begin{array}{l}\text { La melatonina es eficaz en mejorar } \\
\text { la calidad del sueño a corto plazo, } \\
\text { sin embargo no son significativos a } \\
\text { largo plazo ( } 12 \text { meses). }\end{array}$ & EP \\
\hline $\begin{array}{l}\text { Restrepo D, Hidalgo } \\
\text { P, Gomez-Restrepo C, } \\
\text { Gil F, et al; } 2011^{3} \text {. }\end{array}$ & $\begin{array}{l}139 \text { pacientes en } \\
\text { HD. }\end{array}$ & $\begin{array}{l}\text { Presentar una revisión actualizada } \\
\text { de la literatura médica acerca de } \\
\text { los trastornos del sueño en pacien- } \\
\text { tes en diálisis por enfermedad renal } \\
\text { crónica. }\end{array}$ & $\begin{array}{l}\text { Los pacientes en diálisis tienen } \\
\text { alta prevalencia de trastornos del } \\
\text { sueño. Estos afectan negativamen- } \\
\text { te la salud y la calidad de vida de } \\
\text { quien los padece. }\end{array}$ & $\mathrm{RL}$ \\
\hline
\end{tabular}

AHÍ: índice de apnea-hipoapnea; DP: diálisis peritoneal; EP: estudio primario; ERC: enfermedad renal crónica; ERT: enfermedad renal crónica; HD: hemodiálisis; RL: revisión de la literatura; SAOS: síndrome de apnea obstructiva del sueño; SDE: somnolencia diurna excesiva; SPI: síndrome de piernas inquietas.

\section{Síndrome de piernas inquietas y movimiento periódi- co de extremidades}

El síndrome de piernas inquietas (SPI) es una alteración neurosensitiva-motora asociada al sueño, afecta esencialmente a las piernas, cuando se intensifica también puede afectar brazos y otras partes del cuerpo ${ }^{6-11}$.

Por otra parte, el movimiento periódico de extremidades (MPE) es la agitación estereotipada e involuntaria de las extremidades durante el sueño, predominando en las extremidades inferiores ${ }^{12}$.

La diferencia radica en que el movimiento del SPI se expresa en el periodo de vigilia, mientras que el MPE se evidencia en las primeras etapas del sueño (I y II de no REM) $)^{13,14}$.
En relación a los síntomas, el SPI se caracteriza por sensaciones dolorosas en las piernas, simultáneo a una intensa necesidad de moverlas, particularmente durante la noche, aumentan con el reposo y mejora con la actividad, al menos transitoriamente ${ }^{10,11,15,16}$. Mientras que el MPE genera una actividad frecuente y repetitiva del músculo tibial anterior que resulta en el movimiento del ortejo mayor y/o del tobillo, durante el sueño ${ }^{17}$.

La prevalencia de SPI va de $6 \%$ hasta un $60 \%$ en pacientes dializados ${ }^{16-18}$, sin embargo, sus cifras varían según la población, es así que en hemodializados las cifras son cercanas al $40 \%$, mientras que en la población en diálisis peritoneal (DP) van de un $33 \%$ a $50 \% 5,17$, la que varía si la modalidad es automática (APD) o manual (CAPD), encontrando una prevalencia mayor con $A P{ }^{17}$. En cambio, el MPE podría afectar a la población con ERC entre un 40 y 70\% ${ }^{12,17}$. Cabe destacar que 
cerca del $80 \%$ de las personas que tienen SPI, también padecen MPE $3,13,14,20$.

Autores mencionan que la etiología del SPI es desconocida aún ${ }^{11,20-23}$. Sin embargo, existen factores de riesgo que estarían asociados a su aparición, tales como, el déficit de hierro o alteración en su metabolismo, frecuente en embarazadas y en los pacientes con ERC ${ }^{13,14,21}$, lo anterior hace suponer que la anemia desempeña un papel relevante en el desarrollo de SPI ${ }^{11}$. Mortazavi y cols exponen que los síntomas mejoran con agonistas de la dopamina y empeorado con antagonistas dopaminérgicos, por lo que proponen que se relaciona con la disfunción de la dopamina en el sistema nervioso (SN) central ${ }^{14,21}$

En los últimos años se han realizado estudios con el objetivo de encontrar factores de riesgo bioquímicos relacionados con SPI, se estudiaron niveles de ferritina, calcio, fósforo, urea y creatinina; sin embargo, no fueron predictores estadísticamente significativos ${ }^{8,15,24}$. Stefanidis y cols refieren que mujeres, pacientes jóvenes y aquellos con niveles elevados de B2-microglobulina y PTH presentan mayor riesgo de SPI ${ }^{16}$. La duración de cada sesión de HD se asocia a SPI, según Beladi-Mousavi y cols, a mayor tiempo mayor es la intensidad de los síntomas ${ }^{8}$.

EI MPE tiene mayor riesgo a mayor edad de la persona y puede relacionarse con otras comorbilidades, según la guía elaborada por las Sociedades Españolas de Neurología (SEN) y la de Sueño (SES), los síntomas podrían intensificarse con fármacos antidepresivos, situación respaldada por un estudio que incluía polisomnografía en pacientes con antidepresivos frente a pacientes controles, que evidenció que el riesgo de tener MPE era 5 veces mayor en pacientes que tomaban antidepresivos ${ }^{13}$.

Se diagnostica en base de al menos 4 criterios según la guía de "International Restless Legs Syndrome Study Group", entre ellos el deseo de mover los miembros, usualmente asociados con parestesias o disestesias; agitación motora; agravamiento o presentación exclusiva de los síntomas en reposo con alivio total o parcial por actividad y; por último el agravamiento de los síntomas por la noche $e^{8-10,16}$. Considerando que el uso de los criterios podrían causar confusión en el momento de evaluar y aportar falsos positivos, se utilizan métodos diagnósticos complementarios como la polisomnografía y el test de inmovilización sugerida ${ }^{13}$.
En relación al tratamiento farmacológico, está enfocado a los síntomas y no a la causa ni su curso. Entonces, ante la falta de adherencia al fármaco los síntomas no desaparecen. La guía realizada por las SEN-SES, sugiere iniciar con monoterapia en una sola dosis en la más mínima cantidad, 2 o 3 horas antes del comienzo de los síntomas, la dosis podría ir aumentando progresivamente hasta conseguir control sintomático ${ }^{13}$. La evidencia muestra que la primera línea de tratamiento de SPI son los agentes dopaminérgicos y anticonvulsivantes ${ }^{25,26}$. Sin embargo, una revisión de Cochrane concluyó que existe un efecto impreciso de las intervenciones farmacológicas ${ }^{18}$. Por otra parte, existe el tratamiento no farmacológico: masajes, ejercicio, yoga, técnicas de relajación, baños de agua fría o caliente, entre otras.

Además, en ambos es necesario complementarlo con una adecuada higiene del sueño, tomar medidas como dormir lo justo y necesario, evitar siestas y en el caso de pacientes con SPI, retrasar la hora de acostarse para prevenir los movimientos de las extremidades durante el inicio del sueño ${ }^{13}$.

Un estudio con más de 10.000 pacientes, mostró que la actividad física al menos una vez por semana mejoraba la calidad del sueño ${ }^{27}$, sin embargo, a nivel de mejoría de síntomas del SPI en pacientes en diálisis, otros autores no encontraron diferencias estadísticamente significativas si la comparaban con el grupo control. A pesar de los resultados, recomiendan la actividad física aeróbica ${ }^{21}$, así mismo una revisión de Cochrane sugiere que el ejercicio aeróbico podría ser efectivo ${ }^{18}$.

En la misma línea, Giannaki y sus colegas, estudiaron la eficacia de los agonistas dopaminérgicos ante la actividad física, donde ambos fueron efectivos para disminuir la sintomatología del SPI (46\% para el ejercicio y $54 \%$ para el fármaco) y ambas intervenciones mejoraron significativamente la calidad de vida, aunque sólo los agonistas de dopamina mejoraron la calidad del sueño significativamente ${ }^{28}$. Razazian y cols, estudiaron la efectividad de levodopa y de gabapentina en la reducción de síntomas de SPI, éste último mostró ser más efectivo dada la mejora de los síntomas sensitivo-motores del SPI y MPE, con aumento de la calidad del sueño en general ${ }^{29}$.

Respecto a las complicaciones producto del SPI, se mencionan menor supervivencia y disminución en la calidad de vida $6,8,30,31$. Algunos autores refieren que existen mecanismos fisiopatológicos en los que este síndrome predispone a desarrollar enfermedad cardiovascular 
en el paciente en terapia dialítica ${ }^{32,33}$, uno de ellos es el incremento de la actividad del SN simpático y la consecuente hipertensión y taquicardia ${ }^{32}$. Giannaki y cols, sugieren que una menor calidad de vida en pacientes con SPI puede ser por un sueño poco reparador y salud mental alterada ${ }^{30}$. Las complicaciones del MPE se relacionan con un aumento del riesgo cardiovascular en la población dializada versus la general, Lindner refiere que existe una asociación entre el MPE e hipertensión, así como con insuficiencia cardíaca congestiva ${ }^{17}$.

\section{Síndrome de apnea obstructiva del sueño (SAOS)}

EI SAOS es una alteración crónica grave provocada por el colapso frecuente de las vías aéreas superiores que causa asfixia nocturna reiterada e interrupción del sueño. Los repetidos descensos de saturación de oxígeno, incrementan el estrés oxidativo y la activación del SN simpático, esto ocasiona considerables cambios de la presión arterial y ritmo cardiaco ${ }^{7,34-36}$. Las apneas se clasifican en obstructivas, centrales o mixtas; las primeras son causadas por la oclusión intermitente de las vías aéreas, mientras que en las centrales la causa es la ausencia de movimientos respiratorios, producto de una alteración del centro respiratorio. En tanto que la apnea mixta es una mezcla de ambas ${ }^{37}$. En pacientes con ERC predominan las apneas obstructivas, con un leve porcentaje de mixtas o centrales ${ }^{38}$.

Entre sus manifestaciones destaca la interrupción del sueño, que desencadena síntomas durante el día, como la somnolencia diurna excesiva (SDE). Autores refieren que la presentación del SAOS en pacientes con ERC se distingue de la población general por síntomas clásicos, como ronquidos fuertes menos concurrentes, mientras que la hipertensión y fatiga son más frecuentes en pacientes en $\mathrm{HD}^{34,39}$.

Al igual que el SPI, la prevalencia del SAOS en los pacientes dializados es variable y depende de la población estudiada y método diagnóstico utilizado 34,40 . La prevalencia en HD oscila entre un $6 \%$ y $86 \% 6,7,74,40-43$. En tanto que en DP la prevalencia es de un $16 \%$ a un $92 \%$, y si se compara APD y CAPD, los porcentajes son mayores en este último grupo $0^{6,7,41,44,45}$.

Las personas con ERC tienen mayor riesgo de presentar SAOS que la población general, la etiología en el primer grupo se describe como multifactorial y entre los agentes causales se mencionan la sobrecarga de volumen, estrechez de las vías aéreas superiores, inesta- bilidad de la ventilación, edades avanzadas y otras patologías concomitantes como diabetes ${ }^{31,46}$.

Su diagnóstico es difícil de establecer y el mejor método no parece estar definido ${ }^{47}$. Actualmente el gold estándar es la polisomnografía, su alto costo económico limita su implementación en pacientes potencialmente afectados con SAOS $31,34,48$. El criterio diagnóstico también es controvertido, algunas instituciones lo diagnostican con un Índice de apnea e hipoapnea (AHÍ) > a $5 / h^{49}$, mientras la Academia de Medicina del Sueño la considera si es > a 15/h31,34,35,43-45. Además, se utilizan cuestionarios que apoyan el diagnóstico, como la escala de somnolencia de Epworth para evaluar la SDE y el Índice de calidad del sueño Pittsburgh para determinar la calidad subjetiva del sueño $0^{6,7,23,40,43}$. Existen escalas de screening del SAOS para complementar la evaluación, entre los que se destacan "Cuestionario de Berlín", "STOP-BANG Score" y "circunferencia de cuello ajustada" 6, 34,40,48.

La primera línea de tratamiento para el SAOS incluye la modificación del estilo de vida del paciente, pérdida de peso y terapia con presión positiva continua en la vía aérea (CPAP $)^{48}$. Esta última ha mostrado ser efectiva para resolverlo, reduciendo la SDE y mejorando la calidad de vida. Sin embargo, sólo la mitad de los pacientes con indicación de CPAP continúan usándolo a largo plazo, dado que no tienen buena tolerancia o lo rechazan. Sin embargo, una cantidad de personas permanecen con somnolencia a pesar del tratamiento ${ }^{48}$.

Entre las complicaciones del SAOS, se mencionan la SDE, hipertensión, hipertrofia ventricular izquierda, mayor riesgo cardiovascular y por ende, aumento de mortalidad ${ }^{7}, 46,49$. Al respecto, Masuda y cols estudiaron el SAOS como factor de riesgo independiente de mortalidad y sus resultados así lo comprueban ${ }^{50}$.

\section{Somnolencia diurna excesiva}

Es la tendencia de la persona a quedarse dormida durante la vigilia, se reconoce también como la propensión a dormirse o la habilidad de transición de la vigilia al sueño. Es una alteración que evidencia la dificultad de mantenerse en alerta y puede presentarse como manifestación de cualquiera de las patologías mencionadas anteriormente $43,51,52$.

La prevalencia de la SDE varía dependiendo del tipo de diálisis aplicada: en HD oscila entre un $31 \%$ a $77 \%$, en 
tanto que en DP varía de $13 \%$ a $37 \% \%^{7,24}$. Si se realiza un día de diálisis versus un día de no-diálisis, las cifras son mayores en los días de diálisis ${ }^{7}$.

Es causada por alguna deficiencia del sueño, en calidad o cantidad de éste, las más encontradas son el SAOS, insomnio y SPI. Además, se describen factores que determinan su aparición: trastornos neurológicos, psicológicos, pulmonares, presencia de obesidad y/o depresión, edades extremas, anemia, entre otros ${ }^{45,51,52}$.

Para su diagnóstico se utiliza la Escala de Epworth, escala subjetiva de somnolencia, que considera la probabilidad percibida de quedarse dormido en situaciones cotidianas ${ }^{45,48,52}$. Es una prueba fácil de administrar y rápida, sin embargo, depende de la interpretación del evaluador y podría verse afectada por factores sociales, culturales y psicológicos (como ansiedad o depresión), además, puede ser no tan fiable en pacientes muy somnolientos ${ }^{52}$.

El tratamiento de la SDE está enfocado en la causa, complementándose con la promoción de la adecuada cantidad de horas de sueño y en la educación sobre higiene del sueño $0^{52}$. Es importante considerar todos los factores que pudieran influir negativamente en la calidad y cantidad de sueño, esto es fundamental para dar un manejo correcto ${ }^{51}$.

Dentro de las complicaciones se menciona que las actividades cotidianas y calidad de vida son afectadas. Igualmente se asocia a mayores niveles de mortalidad y eventos cardiovasculares ${ }^{10,53,54}$, también contribuye a una disminución cognitiva y alteración de estados anímicos. En pacientes afectados por SDE, existe mayor riesgo de accidentes de tránsito y de trabajo ${ }^{40}$. Algunos autores declaran que existe una reducción en la supervivencia si la calidad del sueño de los pacientes se altera durante el primer año de tratamiento ${ }^{55}$.

\section{Insomnio}

Es la sensación subjetiva de sueño insatisfactorio e insuficiente, aunque se tenga la capacidad para dormir ${ }^{56}$. Puede implicar dificultades para conciliar el sueño, comportamientos infrecuentes del sueño o que éste sea excesivo $^{3-57}$. La Asociación Americana de Centros de Estudio y Tratamientos del Sueño lo define como: "la latencia mayor a 30 minutos $y / 0$ dos o más despertares nocturnos y/o vigilia nocturna mayor a una hora $y / 0$ tiempo de sueño total menor a 6 horas ${ }^{\prime \prime 57}$.
Existen diferentes tipos de insomnio: el insomnio primario o idiopático y el insomnio secundario, que aparece en respuesta a la presencia de un problema médico o mental, abuso de sustancias, alteraciones del sueño 0 higiene del sueño inadecuada ${ }^{58}$.

Cuando el insomnio está presente, por lo general, el paciente manifiesta problemas para conciliar el sueño, despertares frecuentes en la noche o SDE.

Puede clasificarse de acuerdo al momento de presentación: inicial o de conciliación (incremento en latencia del sueño), de mantenimiento (despertares nocturnos con imposibilidad de volver a dormir) o despertar temprano (más temprano que la hora habitual con imposibilidad de volver a dormir $)^{57}$.

Existen diversas variables, incluso por definición y clasificación, que dan origen al amplio rango de prevalencia del insomnio49. En HD las cifras van de un $36 \%$ a $85 \%{ }^{6,56-58}$, entretanto en DP se describe que CAPD es cercana al $81 \%$ y en DPA es $84 \%{ }^{6}$. En un estudio realizado por El Harraqui, la prevalencia de insomnio en sus pacientes fue de $67,7 \%$, lo que demuestra su alta prevalencia ${ }^{58}$.

En particular, los factores de riesgo asociados con el insomnio referidos en la literatura no son pocos y dan cuenta que los pacientes en diálisis tienen más posibilidades de presentarlo, además de objetivarse, mediante polisomnografía, un sueño más corto y poco reparador ${ }^{59}$. Se menciona que la duración de HD mayor a un año es estadísticamente significativa en la aparición del insomnio. Asimismo, existe mayor riesgo en los pacientes de sexo femenino, de edad avanzada, con depresión y/o hipertensión, comorbilidades que impidan el inicio/ mantención del sueño (dolor, disnea, nicturia, etc), BUN alto pre-diálisis, bajos flujos dialíticos, inflamación, poca actividad física y anemia ${ }^{56,58}$.

Los criterios para el insomnio primario son: periodo de latencia $>30$ minutos, tiempo total de vigilia nocturna $>30$ minutos, tiempo total de sueño nocturno menor a 61/2 horas, efectos diurnos (SDE/disminución de rendimiento cognitivo), presentación más de 3 veces semanales, por al menos un mes ${ }^{60}$. El diagnóstico se fundamenta en una anamnesis completa, considerando hábitos de sueño, auto-informe del sueño e información concedida por un tercero. Se deben estudiar características del insomnio: duración, gravedad, causas, características del sueño-vigilia (hora de levantada, periodo de latencia, uso de fármacos o drogas, etc). También es importan- 
te realizar un completo examen físico y psicológico, se puede complementar con polisomnografía, actigrafía y test de latencia del sueño múltiple ${ }^{56,58}$.

El tratamiento del insomnio fue el objeto de estudio de un meta-análisis, con referencia al insomnio primario, ya que en insomnio secundario la resolución radica en tratar la causa principal. Los resultados muestran que terapias cognitivo-conductuales fueron las más utilizadas y las más efectivas. El tratamiento farmacológico es efectivo, aunque su uso es complicado dado que las personas suelen auto-medicarse y quienes acuden al médico, no siempre reciben una prescripción adecua$\mathrm{da}^{61}$. Algunos estudios afirman que el uso de melatonina trae beneficios a corto plazo mejorando parámetros del sueño ${ }^{62,63}$, sin embargo, no se evidencian estos resultados a largo plazo63.

El control de estímulos, restricción del sueño, higiene del sueño y la relajación son consideradas terapias conductuales. Mientras que la imaginación guiada o distracción cognitiva, terapia cognitivo-conductual, aurículo-terapia, biblioterapia y la terapia luminosa son categorizadas como terapias cognitivas ${ }^{61}$.

Las medidas de higiene del sueño se relacionan con el control de factores ambientales y hábitos de salud favorables con el sueño. Por ejemplo, acostarse sólo cuando tenga sueño, levantarse todos los días a la misma hora, no dormir siestas, no consumir cafeína/nicotina de 4 a 6 horas previas a ir a la cama, no beber alcohol en dos horas previas, realizar ejercicio físico siempre y cuando no sea en horas cercanas al acostarse, tener un ambiente cómodo: reducir niveles de luminosidad, de ruido y tener una temperatura agradable ${ }^{60,61}$.

De las intervenciones no farmacológicas, la primera línea son las terapia conductuales, las cuales tienen alta efectividad con cifras que van del $50 \%$ al $80 \%$ de respuesta. Es importante destacar que no se lleva a cabo por sí sola, por lo que se requiere intervenciones combinadas, considerando aspectos del ambiente y la persona ${ }^{61}$.

De acuerdo a los resultados más relevantes del meta-análisis, se categorizaron los diferentes tipos de terapia ${ }^{61}$ :

- Intervención combinada: todas estas intervenciones obtuvieron buenos resultados, que se mantuvieron durante el seguimiento.
- Monoterapias: en esta categoría, los resultados no fueron consistentes: estudios que usaron alguna de las técnicas cognitivas arrojaron leves mejoras, en tanto si se utilizaban otras técnicas como "supresión de pensamientos" o "automonitorización" se aumentaba la latencia de sueño o hubo mayor percepción de deterioro en el funcionamiento.

- Terapia luminosa: todos los estudios que lo aplicaron arrojaron resultados positivos.

- Terapia auricular: es discordante, un estudio mostró leves mejoras que no perduraron en el tiempo y en el otro estudio continuaron hasta seis meses posteriores.

Zou y colaboradores realizaron un ensayo clínico randomizado comparando la acupuntura auricular en puntos específicos versus no específicos para el insomnio en pacientes en $H D$, los resultados fueron favorables para la acupuntura en puntos específicos ${ }^{64}$. En esta línea, Cochrane llevó a cabo una revisión que estima que la evidencia disponible debe ser de mayor calidad para poder recomendar la acupuntura en esta población ${ }^{65}$.

Si se comparan intervenciones farmacológicas versus no farmacológicas, los resultados favorecen a esta última y al combinar ambos tipos de terapia es más efectivo que fármacos por sí solos ${ }^{66}$.

La reducción en la calidad de vida es uno de los efectos del insomnio más mencionados en la literatura ${ }^{58}$. También está asociado con un mayor riesgo de morbi-mortalidad ${ }^{53-54}$, tiene implicancias en diferentes aspectos de la vida: familiar, social y laboral ${ }^{56}$. La depresión y ansiedad pueden ser consideradas causa o efecto del insomnio ${ }^{52}$.

\section{Discusión}

En las personas con ERC que se someten a alguna de las modalidades de diálisis, la prevalencia de trastornos del sueño es alta. Se describen cinco alteraciones que son las más reportadas por la literatura: síndrome de piernas inquietas, movimiento periódico de extremidades, insomnio, síndrome de apnea obstructiva del sueño y somnolencia diurna excesiva. A grandes rasgos, todas influyen negativamente en la calidad de vida del paciente y aumentan la morbimortalidad.

Esta revisión es útil para el equipo de salud y principalmente para enfermeras, ya que acompañan por más tiempo al paciente. Se destaca dentro de sus respon- 
sabilidades el cuidado "integral y holístico", es decir, es imprescindible considerar el ámbito físico, espiritual, psicológico y social de cada una de las personas a su cuidado. Así, a través de la valoración de la persona, reconocer sus necesidades, tomando en cuenta el sueño como una necesidad básica que repercute en su cotidianidad $^{66}$. Ha quedado demostrado que las alteraciones del sueño son un aspecto fundamental en el diario vivir del paciente y que, sin duda, repercute en el deterioro de su calidad de vida. Por ende, es un elemento que el equipo de salud no debiese pasar por alto.

En muchas ocasiones, durante la entrevista inicial el paciente manifiesta que tiene sueño, que no pasó buena noche o que está cansado. Enfermeras tienen la oportunidad de visibilizar esta situación, acoger al paciente y buscar alternativas de solución. Ahora que son conocidas las manifestaciones, los factores de riesgo asociados y el tratamiento farmacológico y no-farmacológico de cada una de las alteraciones del sueño presentes en estos pacientes, es entonces, parte de la responsabilidad de enfermería indagar en la existencia de alteraciones del sueño, sintomatología e intensidad y detectar los signos de los trastornos del sueño. Así como también, sugerir al médico la indicación de fármacos si corresponde, también es relevante que enfermeras puedan recomendar medidas no farmacológicas y educar respecto a ellas, además de los efectos esperados y secundarios de los medicamentos recetados.

Finalmente, con el objetivo de lograr una correcta detección y tratamiento de las alteraciones del sueño, sería importante que instituciones educacionales acreditadas instauren e impartan un programa de capacitación formal sobre higiene del sueño y terapias cognitivo-conductual para la resolución de estos trastornos. Además, es imprescindible que enfermería participe de estas instancias y la aplique en su quehacer cotidiano.

\section{Proyecto: Estudio Independiente de la Escuela de Enfermería, Pontificia Universidad Católica de Chile}

Recibido: 1 julio 2018

Revisado: 5 julio 2018

Modificado: 2 septiembre 2018

Aceptado: 1 noviembre 2018

\section{Bibliografía}

1. Ministerio de Salud. Gobierno de Chile. Guía Clínica Prevención Enfermedad Renal Crónica. Santiago de Chile: Minsal, 2010.

2. Perales Montilla CM, Duschek $S$, Reyes Del Paso GA. Calidad de vida relacionada con la salud en la enfermedad renal crónica: relevancia predictiva del estado de ánimo y la sintomatología somática. Nefrologia. 2016;36(3):275-82.

3. Restrepo D. Trastornos de sueño en los pacientes en diálisis. Rev Colomb Psiquiat 2010;39(3): 588-600.

4. Abdel-Kader K, Jhamb M, Mandich LA, Yabes J, Keene RM, Beach S, et al. Ecological momentary assessment of fatigue, sleepiness, and exhaustion in ESKD. BMC Nephrol 2014;15(1):29.

5. Weisbord SD, Fried LF, Mor MK, Resnick AL, Unruh $M L$, Palevsky PM, et al. Renal provider recognition of symptoms in patients on maintenance hemodialysis. Clin J Am Soc Nephrol. 2007;2(5):960-7.

6. Losso RLM, Minhoto GR, Riella MC. Sleep disorders in patients with end stage renal disease undergoing dialysis: comparison between hemodialysis, continuous ambulatory peritoneal dialysis and automated peritoneal dialysis. Int Urol Nephrol. 2015; 47:369-75.

7. Al-Jahdali H. A comparison of sleep disturbances and sleep apnea in patients on hemodialysis and chronic peritoneal dialysis. Saudi J Kidney Dis Transpl. 2011;22(5):922-30.

8. Beladi-Mousavi SS, Jafarizade $M$, Shayanpour S, Bahadoram M, Moosavian SM, Houshmand G. Restless Legs Syndrome: Associated Risk Factors in Hemodialysis Patients. Nephrourol Mon. 2015 $29 ; 7(6): \mathrm{e} 31967$.

9. Tekdöş Demircioğlu D, Kavadar G, Esen Öre 0̈, Emre TY, Yaka U. Relationship between restless leg syndrome and quality of life in uremic patients. Agri. 2015;27(2):73-8.

10. Wali S0, Alkhouli AF. Restless legs syndrome among Saudi end-stage renal disease patients on hemodialysis. Saudi Med J. 2015 Feb;36(2):204-10. 
11. Salman Y. Restless Legs Syndrome in Patients on Hemodialysis. Saudi J Kidney Dis Transpl. 2011;22(2):368-72.

12. Jung HH, Lee JH, Baek HJ, Kim SJ, Lee JJ. Nocturnal Hypoxemia and Periodic Limb Movement Predict Mortality in Patients on Maintenance Hemodialysis. Clin J Am Soc Nephrol 2010;5(9):1607-13.

13. Sociedad Española del Sueño, Sociedad Española de Neurología. Síndrome de Piernas Inquietas o Enfermedad de Willis-Ekbom. Recomendaciones diagnósticas y terapéuticas del Grupo de Estudio de la Sociedad Española de Neurología y Sociedad Española del Sueño. Madrid: Luzán 5;2013.

14. Clavero P, Rodríguez-Oroz MC. Trastornos del sueño en la enfermedad de Parkinson y otros trastornos del movimiento. Anales Sis San Navarra 2007;30 (Suppl 1):143-53.

15. Rafie S, Jafari M, Azizi M, Bahadoram M, Jafari S. Restless legs syndrome in hemodialysis patients. Saudi J Kidney Dis Transpl 2016 Mar; 27(2):326-30.

16. Stefanidis I, Vainas A, Dardiotis E, Giannaki CD, Gourli P, Papadopoulou D, et al. Restless legs syndrome in hemodialysis patients: An epidemiologic survey in greece. Sleep Med. 2013;14(12):1381-6.

17. Lindner A, Fornadi K, Lazar AS, Czira ME, Dunai $A$, Zoller R, et al. Periodic limb movements in sleep are associated with stroke and cardiovascular risk factors in patients with renal failure. J Sleep Res. 2012;21(3):297-307.

18. Gopaluni S, Sherif $M$, Ahmadouk NA. Interventions for chronic kidney disease-associated restless legs syndrome. Cochrane Database of Systematic Reviews 2016, Issue 11. Art. No.: CD010690. D0I: 10.1002/14651858.CD010690.pub2.

19. National Institutes of Health [Internet]. USA. National Institute of Neurological Disorders and Stroke. 2016. Síndrome de las piernas inquietas. Disponible en: https://espanol.ninds.nih.gov/trastornos/piernas_inquietas.htm

20. Soumeila I, Keita S, Elhassani A, Sidibé M, Alaoui $\mathrm{K}$, Kabbali $\mathrm{N}$ et al. The restless legs syndrome: incidence and risk factors in hemodialysis. Pan Afr Med J. 2015 Jan 13;20:29.
21. Mortazavi M, Vahdatpour B, Ghasempour A, Taheri D, Shahidi S, Moeinzadeh F, et al. Aerobic exercise improves signs of restless leg syndrome in end stage renal disease patients suffering chronic hemodialysis. Sci World J 2013;2013:628142.

22. Gade K, Blaschke $S$, Rodenbeck $A$, Becker $A$, Anderson-Schmidt H, Cohrs S. Uremic Restless Legs Syndrome (RLS) and Sleep Quality in Patients With End-Stage Renal Disease on Hemodialysis: Potential Role of Homocysteine and Parathyroid Hormone. Kidney Blood Press Res 2013;37:458-63.

23. Bastos JP, Sousa RB, Nepomuceno LA, Gutiérrez-Adrianzen $O A$, Bruin $P F$, Araujo $M L$, et al. Sleep disturbances in patients on maintenance hemodialysis: role of dialysis shift. Rev Assoc Med Bras. 2007;53(6):492-6.

24. Al-Jahdali HH, Al-Qadhi WA, Khogeer HA, Al-Hejaili FF, Al-Ghamdi SM, AI Sayyari AA. Restless Legs Syndrome in Patients on Dialysis. Saudi J Kidney Dis Transpl. 2009;20(3):378-85.

25. Philippe R, Kumar N. Restless legs syndrome. Can J Public Heal. 2013;189(6):160527

26. Garcia-Borreguero D, Kohnen $R$, Silber $M H$, Winkelman JW, Earley CJ, Högl B, et al. The longterm treatment of restless legs syndrome/Willis-Ekbom disease: Evidence-based guidelines and clinical consensus best practice guidance: $A$ report from the International Restless Legs Syndrome Study Group. Sleep Med 2013;14(7):675-84.

27. Elder SJ, Pisoni RL, Akizawa T, Fissell R, Andreucci $V E$, Fukuhara $S$, et al. Sleep quality predicts quality of life and mortality risk in haemodialysis patients: results from the Dialysis Outcomes and Practice Patterns Study (DOPPS). Nephrol Dial Transplant. 2008 Mar;23(3):998-1004.

28. Giannaki CD, Sakkas GK, Karatzaferi C, Hadjigeorgiou GM, Lavdas E, Kyriakides T, et al. Effect of exercise training and dopamine agonists in patients with uremic restless legs syndrome: a six-month randomized, partially double-blind, placebo-controIled comparative study. BMC Nephrol. 14:194. doi: 10.1186 / 1471-2369-14-194.

29. Razazian N, Azimi H, Heidarnejadian J, Afshari D, Ghadami MR. Gabapentin versus Levodopa-c for 
the Treatment of Restless Legs Syndrome in Hemodialysis Patients: A Randomized Clinical Trial. Saudi J Kidney Dis Transpl. 2015;26(2):271-8.

30. Giannaki CD, Sakkas GK, Karatzaferi C, Hadjigeorgiou GM, Lavdas E, Liakopoulos V, et al. Evidence of increased muscle atrophy and impaired quality of life parameters in patients with Uremic restless legs syndrome. PLoS One. 2011;6(10):e25180.

31. Mavanur MS, Unruh M. Sleep disordered breathing in patients with chronic kidney disease. Indian $\mathrm{J}$ Med Res. 2010;131:277-84.

32. Stolic R V, Trajkovic GZ, Jekic D, Sovtic SR, Jovanovic AN, Stolic DZ, et al. Predictive Parameters of Survival in Hemodialysis Patients with Restless Leg Syndrome. Saudi J Kidney Dis Transpl. 2014;25(5):974-80.

33. Giannaki C, Zigoulis P, Karatzaferi C, Hadjigeorgiou GM, George KP, et al. Period Limb Movements in Sleep Contribute to Further Cardiac Structure Abnormalities in Hemodialysis Patients with Restless Legs Syndrome. J Clin Sleep Med. 2013 Feb 15; 9(2): 147-53.

34. Forni Ogna V, Ogna A, Pruijm M, Bassi I, Zuercher E, Halabi G, et al. Prevalence and Diagnostic Approach to Sleep Apnea in Hemodialysis Patients: A Population Study. Biomed Res Int. 2015;2015:103686.

35. Ogna A, Forni Ogna $V$, Mihalache A, Pruijm $M$, Halabi G, Phan 0, et al. Obstructive Sleep Apnea Severity and Overnight Body Fluid Shift before and after Hemodialysis. Clin J Am Soc Nephrol 2015;10(6):1002-10.

36. Roumelioti ME, Buysee, DJ, Sanders, MH, Strollo $P$, Newman $A B$, Unruh $M L$, et al. Sleep-disordered breathing and excessive daytime sleepiness in chronic kidney disease and hemodialysis. Clin J Am Soc Nephrol. 2011;6(5):986-94.

37. Cowie MR. Sleep apnea: State of the art. Trends Cardiovasc Med. 2017;27(4):280-89.

38. Beecroft JM, Hoffstein V, Pierratos A, Chan CT, McFarlane PA, Hanly PJ. Pharyngeal narrowing in end-stage renal disease: implications for obstructive sleep apnoea. Eur Respir J Off J Eur Soc Clin Respir Physiol 2007;30(5):965-71.
39. Beecroft J, Pierratos A, Hanly P. Clinical presentation of obstructive sleep apnea in patients with end-stage renal disease. J Clin Sleep Med. 2009 Apr 15;5(2):115-21.

40. Al-Jahdali H. Prevalence of Sleep Apnea and Excessive Day Time Sleepiness in Patients with End-Stage Renal Disease on Dialysis. Saudi J Kidney Dis Transpl. 2012;23(2):251-61.

41. Tuohy CV, Montez-Rath ME, Turakhia M, Chang TI, Winkelman JW, Winkelmayer WC. Sleep disordered breathing and cardiovascular risk in older patients initiating dialysis in the United States: a retrospective observational study using medicare data. BMC Nephrol 2016 Feb 9;17(1):16.

42. Loewen A, Siemens $A$, Hanly P. Sleep disruption in patients with sleep apnea and end-stage renal disease. J Clin Sleep Med 2009;5(4):324-9.

43. Elias RM, Bradley TD, Kasai T, Motwani SS, Chan CT. Rostral overnight fluid shift in end-stage renal disease: Relationship with obstructive sleep apnea. Nephrol Dial Transplant. 2012;27(4):1569-73.

44. Tang SCW, Lam B, Yao TJ, Leung WS, Chu CM, Ho YW, et al. Sleep apnea is a novel risk predictor of cardiovascular morbidity and death in patients receiving peritoneal dialysis. Kidney Int 2010;77(11):1031-8.

45. Tang SCW, Lam B, Lai ASH, Pang CBY, Tso WK, Khong $\mathrm{PL}$, et al. Improvement in sleep apnea during nocturnal peritoneal dialysis is associated with reduced airway congestion and better uremic clearance. Clin J Am Soc Nephrol. 2009;4(2):410-8.

46. Dos Reis I, Danaga AR, de Carvalho I, Fernandes E, Souza I, Julioti J, et al. Cardiovascular risk and mortality in end-stage renal disease patients undergoing dialysis: sleep study, pulmonary function, respiratory mechanics, upper airway collapsibility, autonomic nervous activity, depression, anxiety, stress and quality of life: a prospective, double blind, randomized controlled clinical trial. BMC Nephrol. 2013 Oct 8;14:215.

47. Olivi H. Apnea del Sueño: Cuadro clínico y estudio diagnóstico. Rev Med Clin Las Condes. 2013; 24(3) 359-73. 
48. Roumelioti ME, Argyropoulos C, Buysse DJ, Nayar $H$, Weisbord SD, Unruh ML. Sleep quality, mood, alertness and their variability in CKD and ESRD. Nephron Clin Pract. 2010;114(4):c277-87.

49. Masuda T, Murata M, Honma S, Iwazu Y, Sasaki N, Ogura $M$, et al. Sleep-disordered breathing predicts cardiovascular events and mortality in hemodialysis patients. Nephrol Dial Transplant (2011) 26:228995.

50. Rosales E, Rey de Castro J. Somnolencia: Qué es, qué la causa y cómo se mide. Acta Med Per. 2010; 27(2):137-43.

51. Slater G, Steier J. Excessive daytime sleepiness in sleep disorders. J Thorac Dis 2012;4(6):608-16.

52. Jaber BL, Schiller B, Burkart JM, Daoui $R$, Kraus $M A$, Lee $Y$, et al. Impact of short daily hemodialysis on restless legs symptoms and sleep disturbances. Clin J Am Soc Nephrol. 2011;6(5):1049-56.

53. Restrepo D, Martinez P, Gomez-Restrepo C, Gil F, Castro C. Trastornos del sueño en pacientes en hemodiálisis Prevalencia y características clínicas y epidemiológicas. Rev Colom Psiq 2011;40(3):433-45.

54. Unruh M, Buysee D, Dew MA, Evans I et al. Sleep Quality and Its Correlates in the First Year of Dialysis. Clin J Am Soc Nephrol. 2006;1:802-10.

55. Malaki M, Mortazavi S, Moazemi S, Shoaran M. Insomnia and Limb Pain in Hemodialysis Patients: What is the Share of Restless Leg Syndrome? Saudi J Kidney Dis Transpl. 2012;23(1):15-20.

56. Organización Panamericana de la Salud. Insomnio. Guía de diagnóstico y manejo [Internet], [Consultado 14 junio 2017]; p. 247-57. Disponible en: http:// www.sld.cu/galerias/pdf/sitios/gericuba/guia23.pdf

57. El Harraqui R, Abda N, Bentata Y, Haddiya I. Evaluation and analysis of insomnia in chronic hemodialysis. Pan Afr Med J. 2014 Oct 28;19:221.
58. Anand $\mathrm{S}$, Johansen $\mathrm{KL}$, Grimes $\mathrm{B}$, Kaysen GA, Dalrymple LS, Kutner NG, et al. Physical activity and self-reported symptoms of insomnia, restless legs syndrome, and depression: the comprehensive dialysis study. Hemodial Int 2013;17(1):50-8.

59. Sarrais F, de Castro Manglano P. El insomnio. Anales Sis San Navarra 2007; 30 (Suppl 1):S121-34.

60. Tafoya Ramos SA, Lara Muñoz MC. Intervenciones no farmacológicas en el insomnio primario: la evidencia de los ensayos clínicos controlados en los últimos diez años (1998-2008). Rev Colomb Psiquiatr. $2011 ; 40(2): 310-35$.

61. Koch BC, Nagtegaal JE, Hagen EC, van der Westerlaken MM, Boringa JB, Kerkhof GA, Ter Wee PM. The effects of melatonin on sleep-wake rhythm of daytime haemodialysis patients: a randomized, placebo-controlled, cross-over study (EMSCAP study). $\mathrm{Br} J$ Clin Pharmacol. 2009;67(1):68-75.

62. Russcher $M$, Koch $B C$, Nagtegaal JE, van Ittersum $\mathrm{FJ}$, et al. Long-term effects of melatonin on quality of life and sleep in haemodialysis patients (Melody study): a randomized controlled trial. $\mathrm{Br} \mathrm{J}$ Clin Pharmacol. 2013;76(5):668-79.

63. Zou C, Yang L, Wu Y, Su G, Chen S, Guo X, et al. Auricular acupressure on specific points for hemodialysis patients with insomnia: a pilot randomized controlled trial. Eller K, editor. PLoS One 2015;10(4):e0122724.

64. Kim KH, Lee MS, Kim TH, Kang JW, Choi TY, Lee JD. Acupuncture and related interventions for symptoms of chronic kidney disease. Cochrane Database of Systematic Reviews 2016, Issue 6. Art. No.: CD009440. DOI: 10.1002/14651858.CD009440. pub2

65. García Llana H, Rodríguez Rey R, Trocoli González F, Celadilla Díez 0, Rodríguez Gutiérrez S, Arranz Sánchez $M$ y et al. Consulta de enfermería interdisciplinar de enfermedad renal crónica avanzada: apuntes para un modelo integral de cuidados. Enferm Nefrol 2013;16(2):133-5.

Este artículo se distribuye bajo una Licencia Creative Commons Atribución-NoComercial 4.0 Internacional. https://creativecommons.org/licenses/by-nc/4.0/ 